\title{
Influência da densidade de estocagem no cultivo de juvenis de robalo-flecha mantidos em laboratório(1)
}

\author{
José Jerônimo de Souza-Filho ${ }^{(2)}$ e Vinicius Ronzani Cerqueira ${ }^{(3)}$
}

\begin{abstract}
Resumo - O objetivo deste trabalho foi analisar a influência da densidade de estocagem no crescimento, conversão alimentar e sobrevivência de juvenis de robalo-flecha (Centropomus undecimalis). Os peixes foram coletados em ambiente natural e treinados a aceitar dietas artificiais. Os indivíduos, com comprimento total de $13 \pm 0,4 \mathrm{~cm}$ e peso de $23 \pm 0,3 \mathrm{~g}$ foram estocados em tanques circulares de fibra de vidro de $5 \mathrm{~m}^{3}$, com água do mar e aeração contínua, nas densidades de 3,6 e 9 peixes $/ \mathrm{m}^{3}$, por 180 dias. Os valores de amônia total $(0$ a $0,5 \mathrm{mg} / \mathrm{L})$, temperatura da água $\left(23,3\right.$ a $\left.30,6^{\circ} \mathrm{C}\right)$, salinidade $(17$ a $34 \mathrm{~g} / \mathrm{L}), \mathrm{pH}$ $(7,8$ a 8,4$)$ e oxigênio dissolvido $(4,8$ a $6,9 \mathrm{mg} / \mathrm{L})$ apresentaram padrão similar entre os tratamentos. A conversão alimentar $(1,88,2,06$ e 2,31) e a sobrevivência $(100 \%, 98,9 \%$ e 96,3\%) foram significativamente melhores nos tratamentos com 3 e 6 peixes $/ \mathrm{m}^{3}$. As médias finais de peso $(110,87 \mathrm{e} 80 \mathrm{~g}) \mathrm{e}$ comprimento total $(20,18,5$ e $18 \mathrm{~cm})$ apresentaram diferença significativa. A maior taxa de crescimento foi observada com 3 peixes $/ \mathrm{m}^{3}$; entre as demais não houve diferença. Entretanto, a biomassa final (332, 511 e $703 \mathrm{~g} / \mathrm{m}^{3}$ ) foi diretamente proporcional à densidade. A baixa densidade favorece o cultivo do robalo em relação ao crescimento, sobrevivência e conversão alimentar.
\end{abstract}

Termos para indexação: Centropomus undecimalis, peixe marinho, conversão alimentar, taxa de crescimento, sobrevivência, técnica de criação.

Influence of stocking density on the rearing of juvenile common snook in laboratory

\begin{abstract}
The objective of this work was to evaluate the influence of stocking density on growth, feed conversion ratio and survival of juvenile common snook (Centropomus undecimalis). Fish were collected from the wild and trained to accept artificial diets. Individuals $(13 \pm 0.4 \mathrm{~cm}$ and $23 \pm 0.3 \mathrm{~g}$ ) were stocked into nine 5- $\mathrm{m}^{3}$ circular fiberglass tanks at three different densities: 3,6 and $9 \mathrm{fish} / \mathrm{m}^{3}$ during a period of 180 days. Tanks were supplied with seawater and continuous aeration. Total ammonianitrogen $(0$ to $0.5 \mathrm{mg} / \mathrm{L})$, water temperature $\left(23.3\right.$ to $\left.30.6^{\circ} \mathrm{C}\right)$, salinity $(17$ to $34 \mathrm{~g} / \mathrm{L}), \mathrm{pH}(7.8$ to 8.4$)$ and dissolved oxygen concentration ( 4.8 to $6.9 \mathrm{mg} / \mathrm{L})$ were similar in all tanks. Feed conversion rate $(1.88$, 2.06 and 2.31$)$ and survival $(100 \%, 98.9 \%$ and $96.3 \%)$ were significantly improved for fish stocked at the lower densities $\left(3\right.$ and $\left.6 / \mathrm{m}^{3}\right)$. There were significant differences in average weight $(110,87$ and $80 \mathrm{~g})$ and length $(20,18.5$ and $18 \mathrm{~cm})$ among treatments. Fish stocked at the lowest density $\left(3 / \mathrm{m}^{3}\right)$ showed the highest growth rate, and there was no difference between the others. However, final biomass (332, 511 and $703 \mathrm{~g} / \mathrm{m}^{3}$ ) was directly correlated with density. A low density influences positively growth, survival and feed conversion in common snook rearing.
\end{abstract}

Index terms: Centropomus undecimalis, saltwater fish, feed conversion, growth rate, survival, rearing techniques.

(1) Aceito para publicação em 31 de julho de 2003 .

(2) Bahia Pesca S.A., Av. Adhemar de Barros, 967, Ondina, CEP 40170-110 Salvador, BA. E-mail: oruabo@gd.com.br

(3) Universidade Federal de Santa Catarina, Centro de Ciências Agrárias, Dep. de Aqüicultura, Caixa Postal 476 , CEP 88040-970 Florianópolis, SC. E-mail: vrcerqueira@cca.ufsc.br

\section{Introdução}

A piscicultura marinha no Brasil é uma atividade que ainda não foi disseminada, apesar das excelentes condições naturais, abundância de recursos hídricos e presença de peixes marinhos com extraordinário potencial para a aqüicultura, destacando-se 
os vermelhos Lutjanus spp., garoupas Epinephelus spp., robalos Centropomus spp., tainhas Mugil spp. corvinas da família Scianidae, linguadosParalichthys spp. e pampos da família Carangidae (Benetti \& Feeley, 1998; Muedas \& Vinatea, 1998).

Alguns estudos foram realizados em escala experimental e indicam duas espécies de robalo com boas perspectivas para o cultivo: Centropomus parallelus e C. undecimalis (Patrona, 1984; Tucker Junior, 1987; Pereira et al., 1997; Rodriguez et al., 1998; Barbuio, 1999). Esta última é apontada como a mais promissora, com possibilidades de cultivo intensivo em viveiros de terra, tanques, cercos, tanques-rede ou em cultivo extensivo como controlador biológico (Cerqueira, 1995; Silva, 1996).

Ao longo do desenvolvimento da piscicultura tem-se observado uma tendência ao uso de sistemas de cultivo cada vez mais intensivos. A intensificação dos processos de produção busca alcançar maior produtividade em menores áreas, menor tempo e custo racionalizado (Kubtiza, 1999). Na aqüicultura intensiva, a densidade em que uma espécie pode ser estocada é um importante fator na determinação da viabilidade econômica. Uma maior densidade de estocagem permite um menor custo de produção por peixe, desde que não haja redução substancial na taxa de crescimento e que a sobrevivência seja satisfatória (Björnsson, 1994).

$\mathrm{Na}$ maioria das espécies investigadas, tem sido encontrada uma relação inversa entre densidade de estocagem e taxa de crescimento (Holm et al., 1990; El-Sayed et al., 1995). Contudo, observa-se que a densidade de estocagem mais adequada varia com a espécie, o tamanho comercial, o sistema de criação utilizado e a idade de estocagem dos peixes. Ela é determinada também por fatores exógenos, como temperatura, luz e taxa de alimentação (Wallace et al., 1988). De acordo com Silva \& Siqueira (1997), é importante considerar também a biomassa final projetada e sua relação com a capacidade de suporte do sistema, lembrando que a densidade biológica ótima não será necessariamente a melhor em termos econômicos.

Existem poucos trabalhos envolvendo o efeito da densidade de estocagem no crescimento do robalo em condições intensivas (Higby \& Beulig, 1988; Pereira et al., 1997; Del-Angel et al., 1998; Rodriguez et al., 1998). Portanto, são necessários estudos que ampliem as informações sobre o comportamento dessa espécie em diversas condições de cultivo.

O objetivo deste trabalho foi avaliar a influência da densidade de estocagem no crescimento, conversão alimentar, e sobrevivência de juvenis de robaloflecha.

\section{Material e Métodos}

O experimento foi realizado na Fazenda Experimental de Camarões Oruabo, Bahia Pesca S.A. localizada no Município de Santo Amaro da Purificação, BA (latitude $12^{\circ} 40^{\prime} 28^{\prime \prime} \mathrm{S}$ e longitude $38^{\circ} 44^{\prime} 9^{\prime \prime} \mathrm{O}$ ), de 28/6/1999 a 28/12/1999.

Juvenis selvagens de Centropomus undecimalis Bloch, 1792 (Pisces: Centropomidae) foram capturados no estuário adjacente à fazenda utilizando-se tarrafa e rede de cerco. Os exemplares foram levados ao laboratório onde permaneceram por 30 dias em tanques de $1.000 \mathrm{~L}$. Como medida profilática, no primeiro dia após a captura, adicionou-se em cada tanque formalina comercial (40\%), numa concentração de $25 \mathrm{~mL} / 1.000 \mathrm{~L}$. Após 24 horas a água foi totalmente trocada.

Baseando-se nas recomendações de Kubtiza (1997), os peixes foram adaptados ao alimento inerte com uma sequiência de dietas formuladas (Tabela 1), durante 30 dias. Ao final deste período efetuou-se a biometria inicial. Indivíduos com comprimento total médio de $13 \pm 0,4 \mathrm{~cm}$ e peso médio de $23 \pm 0,3 \mathrm{~g}$ foram distribuídos ao acaso em nove tanques circulares de fibra de vidro, com capacidade de $5.000 \mathrm{~L}$, nas densidades de estocagem de 3, 6 e 9 peixes $/ \mathrm{m}^{3}$, com três repetições. A biomassa inicial média para cada tratamento foi, respectivamente, de 69,3, 136,2 e $204,3 \mathrm{~g} / \mathrm{m}^{3}$. Cada tanque foi abastecido com água bombeada do mar, renovada diariamente em $50 \%$ do volume

Tabela 1. Composição das dietas utilizadas na adaptação dos juvenis do robalo-flecha Centropomus undecimalis ao alimento formulado ${ }^{(1)}$.

\begin{tabular}{lccccc}
\hline Ingrediente & Dieta 1 & Dieta 2 & Dieta 3 & Dieta 4 & Dieta 5 \\
Camarão & $100 \%$ & $75 \%$ & $50 \%$ & $25 \%$ & - \\
Dieta comercial $^{(2)}$ & - & $25 \%$ & $50 \%$ & $75 \%$ & $100 \%$ \\
\hline \multicolumn{7}{c}{ (1)As texturas das dietas 1,2,3, 4 e 5 foram, respectivamente, úmida, úmida, } \\
úmida, semi-úmida e seca. (2) Dieta comercial para alevinos: umidade máxi- \\
ma, 13\%; mínimo de proteína, 48\%; extrato etéreo mínimo, 10\%; máximo de \\
fibras: 6\%; máximo de cinzas, 15\%; máximo de Ca, 3,5\%; mínimo de P, 0,6\%; \\
enriquecimento vitamínico e mineral: vit. A (8.000 UI); vit. D3 (4.000 UI); \\
vit. E (160 mg); vit. K (10 mg); vit. B1 (10 mg); vit. B2 (20 mg); vit. B6 \\
(20 mg); vit. B12 (30 $\mu$ g); niacina (40 mg); ácido fólico (2,0 mg); ácido \\
pantotênico (40 mg); biotina (1 mg); vit. C estabilizada com radicais fosfato \\
e estável ao processo de extrusão (350 mg).
\end{tabular}


total, dispondo de um sistema de aeração individual. A alimentação utilizada durante o experimento teve como referência a dieta 4 do período de adaptação ao alimento inerte, com algumas modificações (Tabela 2). A dieta peletizada foi oferecida à saciedade, duas vezes ao dia ( $8 \mathrm{~h}$ e $17 \mathrm{~h}$ ), todos os dias da semana.

Foram feitas medidas de temperatura (com termômetro de mercúrio) e salinidade (com refratômetro portátil) uma vez por dia, pela manhã (entre 8 h e 9 h). Foram realizadas medições semanais de amônia total, oxigênio dissolvido (com oxímetro digital) e $\mathrm{pH}$ (com peagômetro digital). A amônia total foi determinada por comparação colorimétrica, utilizando um conjunto comercial (kit) para análise de água; o porcentual de amônia não-ionizada foi estimado de acordo com Boyd (1982). A temperatura da água oscilou entre $23,3^{\circ} \mathrm{C}$ e $30,6^{\circ} \mathrm{C}$ e a salinidade entre 17 e $34 \mathrm{~g} / \mathrm{L}$. O oxigênio dissolvido variou entre 4,8 e $6,9 \mathrm{mg} / \mathrm{L}$, correspondendo a $72,7 \%$ e $98,6 \%$ de saturação, respectivamente. A concentração da amônia total variou entre 0,0 e $0,5 \mathrm{mg} / \mathrm{L}$. O maior valor assinalado da amônia não-ionizada $\left(\mathrm{NH}_{3}\right)$ foi de $0,09 \mathrm{mg} / \mathrm{L}$. Os valores de $\mathrm{pH}$ estiveram entre 7,8 e 8,4

Quando detectada a presença de parasitas (copépodes) nos peixes, aplicaram-se banhos de formalina comercial (40\%), a $250 \mathrm{~mL} / 1.000 \mathrm{~L}$ por 30 minutos, realizando-se também a drenagem e assepsia do tanque. Nos três últimos meses do experimento, o tempo de exposição dos peixes à formalina foi de uma hora. No total foram aplicados 12 banhos terapêuticos.

As biometrias foram mensais, abrangendo todos os indivíduos de cada tanque. Os exemplares foram anestesiados com benzocaína a uma concentração de $50 \mathrm{mg} / 1.000 \mathrm{~L}$, medidos ao milímetro mais próximo, utilizando-se ictiômetro graduado, e pesados em balança digital com precisão de $0,1 \mathrm{~g}$.

Tabela 2. Formulação e composição centesimal (matéria seca) da dieta base utilizada durante o experimento com juvenis do robalo-flecha (Centropomus undecimalis).

\begin{tabular}{|c|c|c|c|}
\hline Ingredientes & $\%$ & Composicão & (\%) \\
\hline Dieta comercial triturada ${ }^{(1)}$ & 73,0 & Proteína bruta & 52,7 \\
\hline Camarão fresco & 25,0 & xtrato etéreo & 10,7 \\
\hline Lecitina de soja & 1,0 & Fibra bruta & 1,3 \\
\hline Premix vitamínico-mineral $^{(2)}$ & 0,5 & Cinzas & 12,3 \\
\hline Vitamina $^{(3)}$ & 0.5 & Carboidratos & 23.0 \\
\hline \multicolumn{4}{|c|}{ 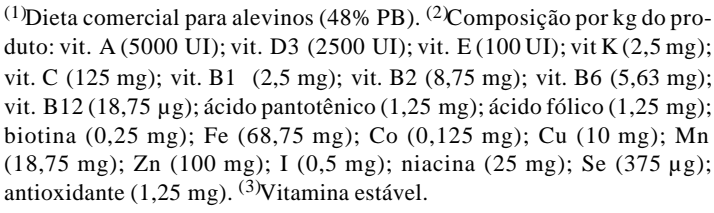 } \\
\hline
\end{tabular}

Além da taxa de sobrevivência foram analisadas as seguintes variáveis: ganho em peso absoluto (peso final peso inicial), ganho em comprimento (comprimento final comprimento inicial), taxa de crescimento específico [(ln peso final - $\ln$ peso inicial)/tempo (dias) x 100] e índice de conversão alimentar (matéria seca do alimento/ganho de peso).

Os dados foram submetidos à análise de variância e as médias comparadas pelo teste de Tukey a 5\% de probabilidade. Os dados de sobrevivência sofreram transformação angular, antes da análise estatística (Pimentel-Gomes, 1987). O coeficiente de variação do peso foi usado como uma medida de variabilidade entre os grupos.

\section{Resultados e Discussão}

A sobrevivência final dos peixes submetidos às densidades de 3 e $6 / \mathrm{m}^{3}$ foi, respectivamente, $100 \%$ e $98,9 \%$, sem diferença significativa entre elas. Na densidade de 9 peixes $/ \mathrm{m}^{3}$ a sobrevivência foi de $96,3 \%$, significativamente inferior às demais (Tabela 3 ).

Não houve ocorrência de canibalismo ou outros problemas que afetassem gravemente a sobrevivência. Entretanto, registrou-se a presença de copépodes do gênero Caligus sp. (Caligidae) fixados à superfície do corpo dos peixes, alimentandose do tecido epitelial. Em determinados casos, verificaram-se graves lesões na pele, ocasionando a penetração de fungos e bactérias, e causando mortalidade em alguns peixes dos tratamentos com 6 e 9 pei$\mathrm{xes} / \mathrm{m}^{3}$. Com o aumento do tempo de banho em formalina, houve redução dos casos de parasitismo.

Tabela 3. Valores (média \pm desvio padrão) de conversão alimentar, sobrevivência, biomassa final, ganho em peso absoluto, ganho em comprimento e taxa de crescimento específico dos juvenis de robalo-flecha (Centropomus undecimalis) após 183 dias do início do experimento ${ }^{(1)}$.

\begin{tabular}{lccc}
\hline Variáveis & \multicolumn{3}{c}{ Densidade (peixes $\left./ \mathrm{m}^{3}\right)$} \\
\cline { 2 - 4 } & \multicolumn{1}{c}{6} & 9 \\
\hline Conversão alimentar & $1,88 \pm 0,11 \mathrm{a}$ & $2,06 \pm 0,03 \mathrm{a}$ & $2,31 \pm 0,05 \mathrm{~b}$ \\
Sobrevivência $(\%)$ & $100 \mathrm{a}$ & $98,9 \pm 1,2 \mathrm{a}$ & $96,3 \pm 1,2 \mathrm{~b}$ \\
Biomassa final $\left(\mathrm{g} / \mathrm{m}^{3}\right)$ & $332,4 \pm 4,1 \mathrm{a}$ & $511,5 \pm 2,7 \mathrm{~b}$ & $703,7 \pm 5,9 \mathrm{c}$ \\
Ganho de peso $(\mathrm{g})$ & $87,7 \pm 3,1 \mathrm{a}$ & $63,5 \pm 3,8 \mathrm{~b}$ & $58,5 \pm 2,8 \mathrm{~b}$ \\
Ganho em & & & \\
comprimento & $(4)$ & & \\
Taxa de crescimento & $7,6 \pm 0,3 \mathrm{a}$ & $5,5 \pm 0,2 \mathrm{~b}$ & $5,2 \pm 0,3 \mathrm{~b}$ \\
específico & $(\%)$ & & \\
\hline
\end{tabular}

(1) Médias seguidas pela mesma letra, na linha, não diferem entre si pelo teste de Tukey a $5 \%$ de probabilidade. ${ }^{(2)}$ Matéria seca do alimento/ganho de peso. ${ }^{(3)}$ Peso final - peso inicial. ${ }^{(4)}$ Comprimento final - comprimento inicial. ${ }^{(5)}$ (ln peso final - ln peso inicial)/tempo (dias) x 100. 
Perdas econômicas em cultivos de várias espécies de peixes, causadas por caligídeos, têm sido relatadas em diferentes partes do mundo. Laviña (1978) verificou a ocorrência de mortalidade do peixe-leite, Chanos chanos, infectado por Caligus patulus, em viveiros estuarinos nas Filipinas. Similarmente, Paperna \& Lahav (1974) relataram a mortalidade de Mugil cephalus em conseqüência dos efeitos do parasitismo por Pseudocaligus apodus, em Israel.

Apesar dos problemas constatados com o parasitismo e manipulação freqüente dos robalos, ocasionada pelos banhos terapêuticos, a elevada sobrevivência em todos os tratamentos indicou um fácil condicionamento dos juvenis desta espécie às condições de confinamento.

As médias finais de peso, 110, 87 e $80 \mathrm{~g}$, respectivamente, nas densidades de 3,6 e 9 peixes $/ \mathrm{m}^{3}$, demonstraram que o tratamento com menor densidade foi superior aos demais (Figura 1). Essa tendência foi observada em relação ao ganho em comprimento e taxa de crescimento específico. Por sua vez, a biomassa final obtida na densidade de 9 peixes $/ \mathrm{m}^{3}$ foi superior aos valores das demais densidades ( $\mathrm{Ta}$ bela 3). Vale ressaltar que este resultado, a relação
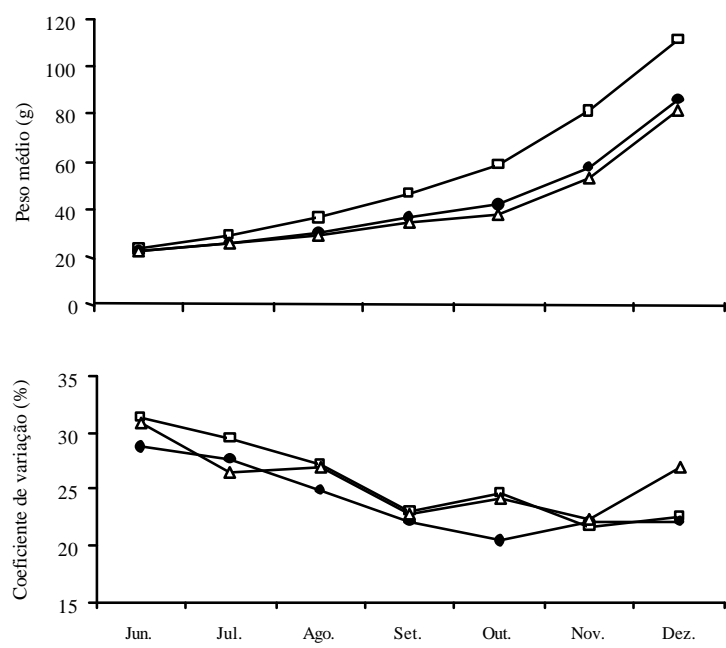

Figura 1. Curva de crescimento em peso e coeficiente de variação do peso de juvenis do robalo Centropomus undecimalis, submetidos às densidades de estocagem de $3(\square), 6(\mathbf{O})$ e $9(\Delta)$ peixes $/ \mathrm{m}^{3}$ durante o período experimental. direta entre densidade e produção de biomassa, é o mais interessante em termos de piscicultura. Todavia, houve uma relação inversa entre taxa de crescimento e densidade de estocagem, o que está de acordo com outros estudos realizados com esta espécie.

Assim, Rodriguez et al. (1998) demonstraram que C. undecimalis, cultivado em tanques-rede fixos, submetido às densidades de 7,5 e 12,5 peixes $/ \mathrm{m}^{3}$, apresentou melhor crescimento na menor densidade testada, ao término de 105 dias. Del-Angel et al. (1998), avaliando o crescimento de $C$. undecimalis em viveiros de terra nas densidades de 0,25, 0,5 e 1 indivíduo $/ \mathrm{m}^{2}$, constataram que, após 118 dias, o melhor resultado de crescimento foi obtido na densidade de 0,25 peixe $/ \mathrm{m}^{2}$.

Esses dados são condizentes com os relatados para muitas espécies de peixes cultivados. El-Sayed et al. (1995) constataram que o melhor desenvolvimento de juvenis do macua-de-pintas-brancas, Siganus canaliculatus, foi alcançado com a menor densidade de estocagem utilizada. Em estudos realizados com a truta arco-íris, o melhor crescimento também foi obtido com a diminuição da densidade de estocagem (Holm et al., 1990).

Wallace et al. (1988) encontraram resultados diferentes ao estudar Salvelinus alpinus, ou seja, a maior média de peso foi obtida na população com maior densidade de estocagem. De forma semelhante, Papoutsoglou et al. (1998), estudando o robalo europeu Dicentrarchus labrax, concluíram que altas taxas de estocagem podem provocar o desenvolvimento do comportamento de aprendizagem, ao mesmo tempo em que diminuem a formação de dominância hierárquica, territorialismo e agressividade entre os peixes.

Alguns estudos têm sugerido que um aumento no coeficiente de variação seja indicativo do estabelecimento de dominância hierárquica e competição pelo alimento, com a supressão do crescimento de indivíduos subordinados por peixes dominantes (Higby \& Beulig, 1988; Bagley et al., 1994). Neste trabalho, em todas as densidades, houve redução do coeficiente de variação e o tratamento com maior valor final foi o de 9 peixes $/ \mathrm{m}^{3}$ (Figura 1). Assim, com base neste parâmetro, não parece ter havido dominância hierárquica associada à variação de densidade. Entretanto, um padrão de comportamento 
agressivo foi observado em todos os tratamentos quando alguns peixes, prestes a abocanhar o alimento, desistiam diante da presença de um outro que se aproximava. Tal conduta era acompanhada, em certos casos, de ataques (perseguição com tentativa de morder o oponente).

Em relação à conversão alimentar, o tratamento com densidade de 9 peixes $/ \mathrm{m}^{3}$ foi significativamente inferior aos demais (Tabela 3). Os valores observados estiveram dentro das faixas relatadas em outros estudos com espécies do gênero Centropomus (Tucker Junior, 1987; Del-Angel et al., 1998; Rodriguez et al., 1998; Barbuio, 1999). De acordo com Vijayan \& Leatherland (1988), a taxa de crescimento dos peixes é dependente da alimentação e da conversão alimentar. Há situações em que esses processos podem ser prejudicados, com uma resposta ao estresse associado com altas densidades de estocagem, afetando as taxas de crescimento. A conversão alimentar foi desfavorecida pelo aumento da densidade de estocagem, porém não houve diferença significativa entre as duas menores densidades $\left(3 \mathrm{e} 6 / \mathrm{m}^{3}\right)$. A conversão alimentar menos favorável com 9 peixes $/ \mathrm{m}^{3}$ poderia ser uma resposta fisiológica para a condição de densidade mais elevada, o que explica a menor taxa de crescimento deste tratamento.

\section{Conclusões}

1. A taxa de crescimento, a sobrevivência e a conversão alimentar do robalo-flecha são influenciadas pela densidade de estocagem, com melhor performance do cultivo na menor densidade.

2. A biomassa final obtida é diretamente proporcional à densidade.

\section{Agradecimentos}

Aos colegas da Bahia Pesca S.A., pelo apoio a este projeto; ao Prof. César Carqueija, da Universidade Federal da Bahia, pela identificação dos copépodes parasitas; aos Drs. Evoy Zaniboni Filho e Débora Machado Fracalossi, da Universidade Federal de Santa Catarina, pela revisão da dissertação de mestrado que deu origem a este trabalho.

\section{Referências}

BAGLEY, M. J.; BENTLEY, B.; GALL, G. A. E. A genetic evaluation of the influence of stocking density on the early growth of rainbow trout (Oncorhynchus mykiss). Aquaculture, Amsterdam, v. 121, p. 313-326, 1994.

BARBUIO, M. A. T. Efeito da utilização de uma dieta comercial e dietas experimentais, nas formas seca e semi-úmida, no crescimento e composição corporal do robalo (Centropomus parallelus Poey, 1860). 1999. 57 f. Dissertação (Mestrado em Aqüicultura) - Universidade Federal de Santa Catarina, Florianópolis, 1999.

BENETTI, D. D.; FEELEY, M. W. Recent progress in marine fish aquaculture. In: SIMPÓSIO BRASILEIRO DE AQÜICULTURA, 10.; CONGRESSO SUL-AMERICANO DE AQÜICULTURA, 1.; SIMPÓSIO BRASILEIRO SOBRE CULTIVO DE CAMARÃO, 5., 1998, Recife. Anais... Recife: Associação Brasileira de Aqüicultura, 1998. p. 9-57.

BJÖRNSSON, B. Effects of stocking density on growth rate of halibut (Hippoglossus hippoglossus) reared in large circular tanks for three years. Aquaculture, Amsterdam, v. 123, p. 259-270, 1994.

BOYD, C. E. Water quality management for fish culture. Amsterdam: Elsevier Scientific, 1982. 730 p.

CERQUEIRA, V. R. Observações preliminares sobre o crescimento de juvenis de robalo Centropomus parallelus e Centropomus undecimalis, com dietas naturais e artificiais. In: CONGRESSO DE ENGENHARIA DE PESCA, 7., 1991, Santos. Anais... Recife: Associação dos Engenheiros de Pesca, 1995. p. 85-94.

DEL-ANGEL, L. E. A.; RODRIGUEZ, P. C.; MENDOZA, G. E. G.; CRESWELL, R. L. Common snook culture Centropomus undecimalis (Bloch, 1792) in earthen freatic ponds in Isla del Carmen, Campeche, Mexico. In: ANNUAL MEETING OF THE GULF AND CARIBBEAN FISHERIES INSTITUTE, 50., 1997, Mérida. Proceedings... Fort Pierce: Harbor Branch Oceanographic Institution, 1998. p. 513-523.

EL-SAYED, A. F. M.; MOSTAFA, K. A.; ALMOHAMMADI, J. S.; EL-DEHAIMI, A.; KAYID, M. Effects of stoking density and feeding levels on growth rates and feed utilization of rabbitfish Siganus canaliculatus. Journal of the World Aquaculture Society, Baton Rouge, v. 26, n. 2, p. 212-216, 1995.

HIGBY, M.; BEULIG, A. Effects of stocking density and food quantity on growth of young snook, Centropomus 
undecimalis, in aquaria. Florida Scientist, Tampa, v. 51, n. 3/4, p. 161-171, 1988.

HOLM, J. C.; REFSTIE, T.; BO, S. The effect of fish density and feeding regimes on individual growth rate and mortality in rainbow trout (Oncorhynchus mykiss). Aquaculture, Amsterdam, v. 89, p. 225-232, 1990.

KUBTIZA, F. Preparo de rações e estratégias de alimentação no cultivo intensivo de peixes carnívoros. In: SIMPÓSIO INTERNACIONAL SOBRE NUTRIÇÃO DE PEIXES E CRUSTÁCEOS, 1995, Campos do Jordão. Anais... Campinas: Colégio Brasileiro de Nutrição Animal, 1997. p. 91-115.

KUBTIZA, F. Tanques-rede, rações e impacto ambiental. Revista Panorama da Aqüicultura, Rio de Janeiro, v. 9, n. 51, p. 44-50, 1999.

LAVIÑA, E. M. A study on certain aspects on the biology and control of Caligus sp.: an ectoparasite of the adult milkfish Chanos chanos (Forskaal). Fisheries Research Journal of the Philippines, Los Baños, v. 3, n. 2, p. 11 24,1978

MUEDAS, W. L.; VINATEA, L. A. Una crítica al modelo económico de desarrollo de la acuicultura Latinoamericana. In: SIMPÓSIO BRASILEIRO DE AQÜICULTURA, 10.; CONGRESSO SUL-AMERICANO DE AQÜICULTURA, 1.; SIMPÓSIO BRASILEIRO SOBRE CULTIVO DE CAMARÃO, 5., 1998, Recife. Anais... Recife: Associação Brasileira de Aqüicultura, 1998, p. 9- 57

PAPERNA, I.; LAHAV, M. Mortality among grey mullets in a seawater pond due to caligid parasitic copepod epizootic. Israeli Journal of Aquaculture - Bamidgeh, Nir David, v. 26, p. 12-15, 1974.

PAPOUTSOGLOU, S. E.; TZIHA, G.; VRETTOS, X.; ATHANASIOU, A. Effects of stocking density on behavior and growth rate of European sea bass (Dicentrarchus labrax) juveniles reared in closed circulated system. Aquacultural Engineering, Amsterdam, v. 18, p. 135-144, 1998.

PATRONA, L. D. Contribution à la biologie du "robalo" Centropomus parallelus (Pisces Centropomidae) du Sud-Est du Brésil: possibilités aquacoles. 1984. 175 f. Thèse (Doctorat de $3^{\text {me }}$ Cycle, Sciences et Techniques en Productions Animales) - Institut National Polytechnique de Toulouse, France, 1984.

PEREIRA, J. A.; SANTOS, G. A. C.; MACEDO, S. J.; SANTANA, M. F. A. Monocultivo do camurin, Centropomus undecimalis (Bloch, 1792) em viveiros-rede, Pernambuco (Brasil). Trabalhos Oceanográficos da UFRPE, Recife, v. 25, p. 61-68, 1997.

PIMENTEL-GOMES, F. A estatística moderna na pesquisa agropecuária. 3. ed. Piracicaba: Potafos, 1987. $162 \mathrm{p}$.

RODRIGUEZ, P. C.; DEL-ANGEL, L. E. A.; CRESWELL, R. L. Growth of common snook, Centropomus undecimalis (Bloch, 1972), in fixed cages in the Pom Lagoon, Campeche, Mexico. In: ANNUAL GULF AND CARIBBEAN FISHERIES INSTITUTE, 50., 1997, Mérida. Proceedings... Fort Pierce: Harbor Branch Oceanographic Institution/ Gulf and Caribbean Fisheries Institute, 1998. p. 524-535.

SILVA, A. L. N. Tilápia vermelha (híbrido de Oreochromis spp.) e camorim Centropomus undecimalis (BLOCH, 1792): aspectos biológicos e cultivo associado na região nordeste do Brasil. 1996. 200 f. Tese (Doutorado em Ecologia e Recursos Naturais) - Universidade Federal de São Carlos, São Carlos, 1996.

SILVA, A. L. N.; SIQUEIRA, A. T. Piscicultura em tanques-rede: princípios básicos. Recife: Sudene/Imprensa Universitária da UFRPE, 1997. 72 p.

TUCKER JUNIOR, J. W. Snook and tarpon snook culture and preliminary evaluation for commercial farming. Progressive Fish-Culturist, Bethesda, v. 49, p. 49-57, 1987.

VIJAYAN, M. M.; LEATHERLAND, J. F. Effect of stocking density on growth and stress-response in brook char, Salvelinusfontinalis . Aquaculture, Amsterdam, v. 75, p. 159-170, 1988.

WALLACE, J. C.; KOLBEINSHAVN, A. G.; REINSNES, $\mathrm{T}$. The effects of stocking density on early growth in artic char, Salvelinus alpinus (L.). Aquaculture, Amsterdam, v. 73, p. 101-110, 1988. 